\title{
Outcomes from Different Minimally Invasive Approaches for Infected Necrotizing Pancreatitis
}

\author{
Yong $\mathrm{Hu}^{1}$, Xun Jiang ${ }^{1}$, Chunyan $\mathrm{Li}^{1}$ and Yunfeng Cui*2 \\ ${ }^{1}$ Tianjin Medical University, Heping District, China \\ ${ }^{2}$ Department of Surgery, China \\ *Corresponding author: Yunfeng Cui, Department of Surgery, Tianjin 300100, China
}

\begin{tabular}{l}
\hline ARTICLE INFO \\
\hline Received: 幽 May 05, 2019 \\
Published: ${ }^{-4}$ May 16, 2019 \\
\hline Citation: Yong Hu, Xun Jiang, Chunyan \\
Li, Yunfeng Cui. Outcomes from Dif- \\
ferent Minimally Invasive Approaches \\
for Infected Necrotizing Pancreatitis. \\
Biomed J Sci \& Tech Res 18(1)-2019. \\
BJSTR. MS.ID.003100.
\end{tabular}

Keywords: Infected Necrotizing Pancreatitis; Minimally Invasive Surgery; Primary Endpoints; Secondary Endpoints

\section{ABSTRACT}

Background: Infected necrotizing pancreatitis (INP), the leading cause of mortality in the late phase of acute pancreatitis (AP), nearly always requires intervention. In recent years minimal invasive surgery are becoming more and more popular for the management of INP, but few studies compared different minimally invasive strategies. The objective of this observation study was to evaluate the safety and efficacy with several minimal invasive treatment.

Materials and Methods: We retrospectively reviewed cases of percutaneous catheter drainage (PCD), minimal access retroperitoneal pancreatic necrosectomy (MARPN), small incision pancreatic necrosectom (SIPN), single-incision access port retroperitoneoscopic debridement (SIAPRD) for INP between January 2013 and October 2018. Data were analysed for the primary endpoints as well as secondary endpoints.

Results: 81 patients with INP were treated by minimally invasive procedures including PCD $(n=32)$, MARPN $(n=18), \operatorname{SIPN}(n=16)$ and SIAPRD $(n=15)$. Overall mortality was greatest after PCD $34 \%$ ( MARPN 11\% vs SIPN 6\% vs SIRLD6\%).Problems after initial surgery were ongoing sepsis (PCD 56\% vs MARPN 50\% vs SIPN 31\%vs SIAPRD13\%; P<0.05).There was a significant difference in number of interventions (median, 6vs5vs3vs2; $\mathrm{P}<0.05$ ). Time from onset of symptoms to recovery was less for SIAPRD than for PCD, MARPN or SIPN (median, 45vs102vs80vs67days; $\mathrm{P}<0.05$ ).

Conclusion: SIAPRD remedy evidently improved outcomes, including SIRS, number of interventions, LOS and overall cost. It is technically feasible, safe, and effective for INP, in contrast to others, and can achieve the best clinical results with the least cost. Furthermore, relevant multicentre RCTs are eager to prove these findings.

\section{Introduction}

Acute necrotic collections (ANC) and walled-off necrosis (WON), often performed at least two weeks after the onset of symptoms [1], become infected in about one-third of patients [2]. It is associated with higher mortality rate of up to $30 \%$ and an acknowledged indication for surgical intervention [3]. It occurs only in moderately severe or severe AP and remains a major public health burden with over 300,000 hospitalizations/year in the United States, accounting for the second highest cost of hospital stays (2.5 billion dollars) [4]. Early aggressive fluid resuscitation, enteral nutrition, antibiotics and intervention are of vital importance to treat INP [5]. Surgical methods and timing are the focus of controversy in the treatment of INP [6]. Traditionally, laparotomy was the only tool available for surgical treatment of pancreatic necrosis [7]. But several clinical evidence was found to be associated with high rate of prolonged multi organ failure, mortality and result in local complications such as bleeding, gastrointestinal fistula, reoperations, as well as a high rate of post-operative diabetes, mainly due to the deterioration of general condition and the serious damage to the abdominal structure and pancreatic tissue [8]. Therefore, minimal invasive techniques have been developed to reduce surgical stress, and 
thereby limit its deleterious influence on patient's condition. The use of minimally invasive techniques, such as PCD and MARPN has gained increasing popularity in a few medical centres [9].

At present, most scholars believe that the intervention should be delayed to about 4 weeks later, and more clinical evidence is needed to confirm it. Several cohort studies on necrotizing pancreatitis have been published over the past decades, 88 patients reported in Hjalmar C's RCT underwent minimally invasive approach which reduced the rate of major complications or mortality compared with open surgery [10]. 98 patients included in van Brunschot's RCT show neither major complications nor mortality differed between the two groups, although fistulas were less common and hospital stays were shorter in the endoscopy arm [11]. Although these studies were methodologically sound, they included highly selective patients, most of whom had no organ failures. In clinical practice, organ failures are common in patients with IPN. In short, minimally invasive approach being more preferable than open surgery has become an expert consensus, but the best of minimally invasive interventions is currently not cleared. Over the past 5 years, our experienced pancreatic multidisciplinary group has embraced several novel minimally invasive approaches to NP treatment with great breakthroughs. In order to evaluate the best operation, we conducted a retrospective study of the patients treated in our surgery centre, by comparing the treatment results with the various surgical intervention, hoping to find out the most ideal minimally invasive approach in INP.

\section{Patients and Methods}

81 consecutive patients with a diagnosis of INP admitted to Nankai University Nankai Hospital who underwent either PCD, MARPN, SIPN or SIAPRD between January 2013 and October 2018 were identified retrospectively and included in this cohort analysis. Twenty-nine cases had been treated at outside facilities for 4-30 days but their condition gradually deteriorated and was complicated by hypovolemia, hypoxemia, and high fever, so they were transferred to our hospital. Exclusion criteria were postsurgical acute pancreatitis, acute pancreatitis as a secondary diagnosis, and the lack of clinical and laboratory data. The study was approved by the Ethics Committee of the Nankai University Nankai Hospital (NKYY_YX_IRB_2018_002_01). Written informed consent was obtained from all patients or their parents prior to surgery. AP was defined according to the 2013 revision of the Atlanta classification as an association of two of the three following features: typical abdominal pain (acute onset of a persistent, severe, epigastric pain often radiating to the back), serum lipase or amylase activity at least three times greater than the upper limit of normal, and characteristic findings of acute pancreatitis on abdominal cross-sectional imaging studies [12]. INP has a mortality of $30 \%$, which can be diagnosed in three ways:

a) By gas configurations in the necrotic collection on imaging

b) By a positive gram stain or culture from a (percutaneous) fine-needle aspiration of the necrotic collection or

c) Suspected by clinical diagnosis.

Clinical suspicion of infection is based on signs of infection (temperature $>38.5^{\circ} \mathrm{C}$, rising serum inflammatory markers) or when new/persistent organ failure occurs, which is typically most reliable after the initial phase of SIRS [13]. Sepsis was defined according to the 1992 ACCS/SCCM criteria [14]. A contrast-enhanced abdominal CT scan showed the area of infected necrosis, including the lesser sac, left or right anterior pararenal space, retroduodenal space, and left or right paracolic gutters. The electronic records of all patients treated at our institution were searched for the ICD code of acute pancreatitis from January 2013 to October 2018 so as not to miss any patients with INP. All patients who underwent minimally invasive treatment were identified by OPS codes and patient records reviewed. For analysis, 4 groups were defined: PCD, MARPN, SIPN, and SIAPRD.

\section{Surgical Protocol}

MARPN: Under general anesthesia, the catheter is exchanged over a guide wire for serial renal dilators and the track dilated to 30F. An operating choledochoscope or nephroscope with a widebore operating channel (initially Wolf, later Storz) is then used to access the necrosis, and if necessary, a combination of softmirror and hard-lens was used. Normal saline is quickly flushed into the pus cavity through the water injection hole, and pus is vacuumed out by negative pressure suction function repeatedly. If solid necrotic tissue attachment is found on the wall of pus cavity under video, it can be removed by piecemeal with videoassisted biopsy forceps. Samples of the removed necrosis are sent for microbiological examination. Following initial debridement, a multifunctional irrigating drain (M10), consisting of a porous outer sleeve and an inner core that can be attracted by vacuum, is inserted into the cavity and $0.9 \%$ saline solution used to irrigate the cavity continuously at a rate of $125 \mathrm{~mL} / \mathrm{h}$ (Figure 1). 

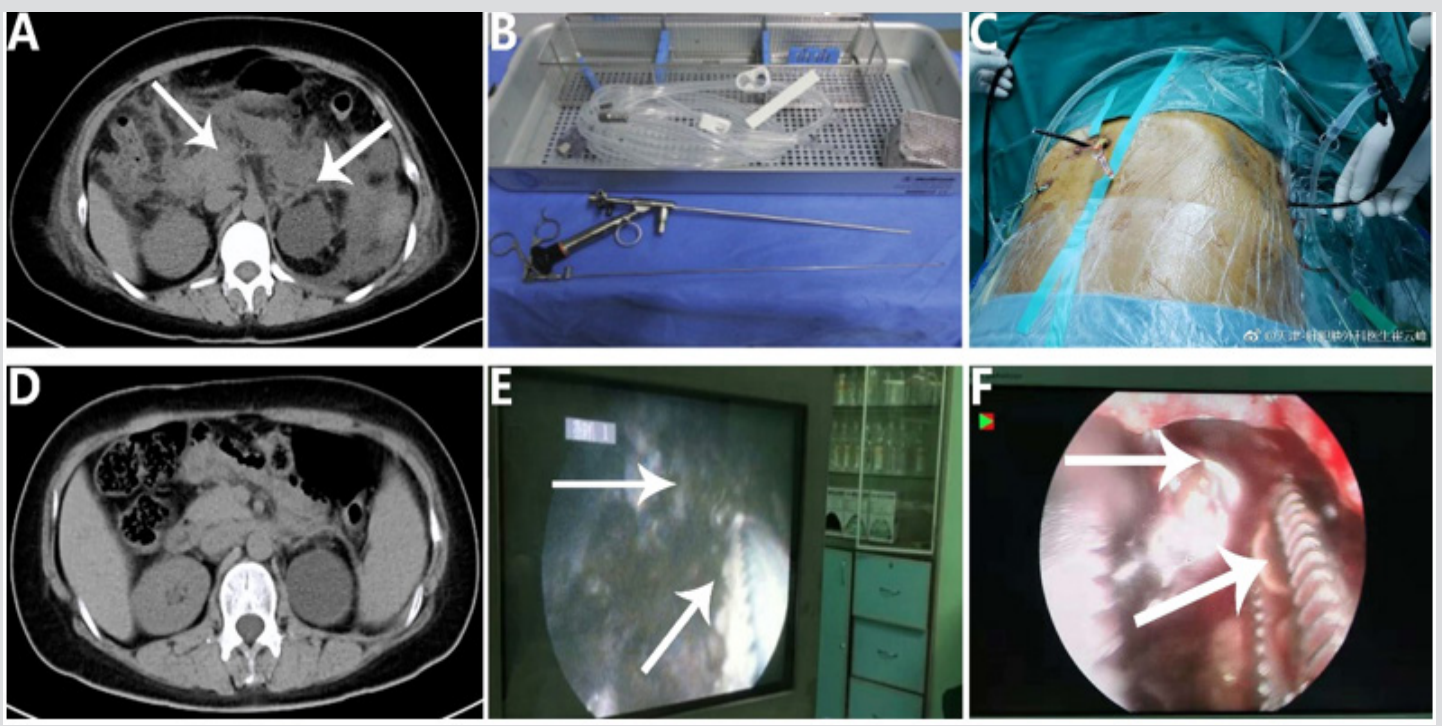

Figure 1: A: computed tomography before the first necrosectomy; B, E: percutaneous nephroscopic device and video-guided necrotic tissue removal; C, F: Cholangioscopy-guided necrotic tissue removal; D: computed tomography after last necrosectomy.

SIPN: The position of the access was determined under CT guidance. Generally, when the infection necrosis area is located at the bilateral retroperitoneal space of the colon, incision is carried out near the mid-axillary line of the bilateral lumbocostal region, while the necrotic infection area is located in the peripancreatic space and the lesser omental sac, the nearest point to the skin is taken for incision. Make a small incision of about $2-5 \mathrm{~cm}$ at the abdominal wall of the drainage tube, and dissect the skin, subcutaneous, muscle and fascia layer by layer. During the whole process, the sinus path formed by the PCD drainage tube was used to enter the abscess cavity (which could greatly reduce the possibility of gastrointestinal side-injury caused by the mistake into the abdominal cavity). A large number of necrotic tissues within the cavity were found under direct vision and then removed with non-invasive forceps or manual. The peripancreatic collection is reached through the space between the spleen, the left kidney and the descending colon. The peripancreatic area is accessed by pushing aside the posterior parietal peritoneum and the colon towards the midline, taking the left kidney as a reference. The necrotic tissue was removed and then the pus cavity was washed with normal saline to confirm that there was no residual necrotic tissue. As far as possible, multiple multifunctional flushing drainage tubes (M10) were inserted into the cavity and removing necrotic tissue by positive pressure irrigation with $0.9 \%$ saline solution and continuous negative pressure suction. If there are multiple infectious foci of pancreatic necrosis, multiple mini incisions can be used to debride necrotic tissue (Figure 2).
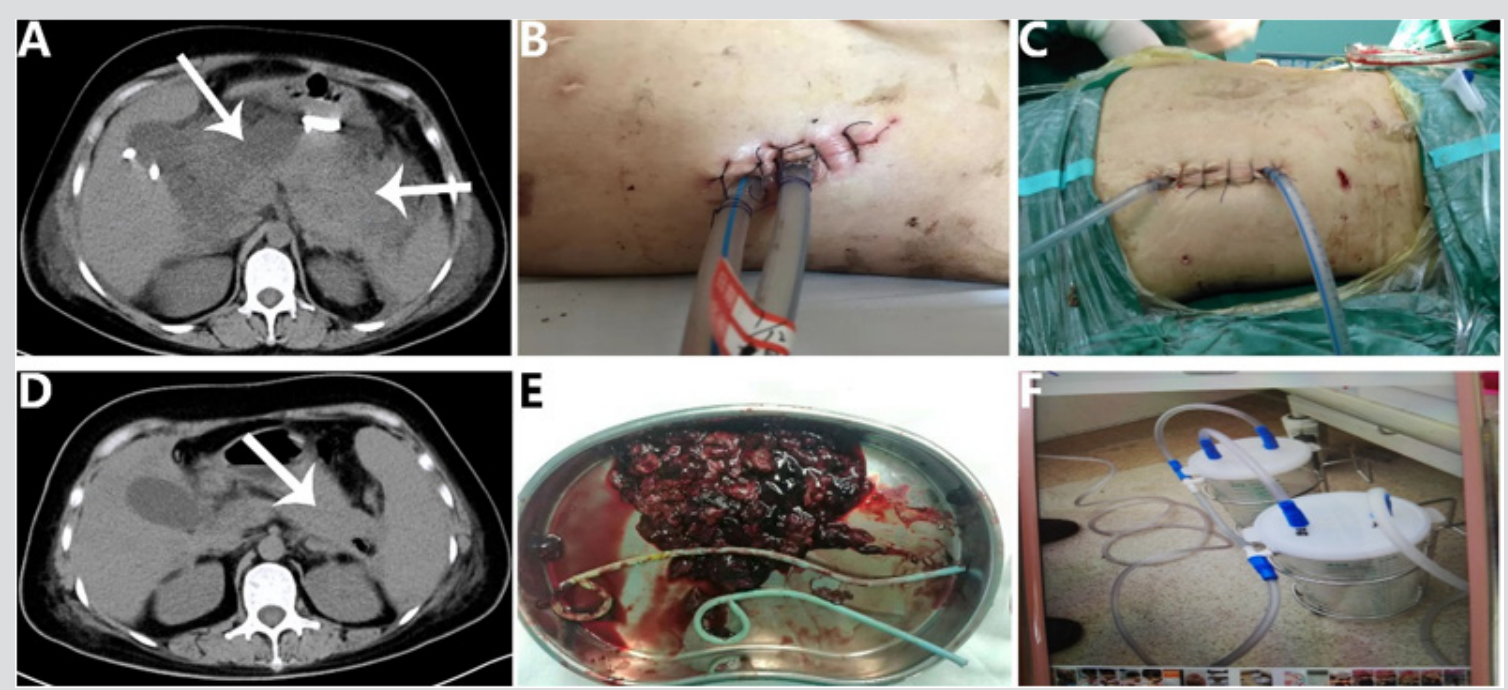

Figure 2: A: computed tomography before the first necrosectomy; B, C: small incision minimally invasive approach; D: computed tomography after last necrosectomy; E: infected necrotic tissue; F: negative pressure flushing device. 
SIAPRD: Under general anesthesia, we use a single access port placed through a short incision in the left lumbar region. The patient was placed in the lateral position $\left(60^{\circ}\right)$ with the affected side facing upward and fixed with position frames. Necrotic collections are accessed through the left retroperitoneum with a small incision of about $2-3 \mathrm{~cm}$ at the abdominal wall of the drainage tube mouth, establish pneumoperitoneum by pneumoperitoneal needle puncture. This port is a flexible soft foam device with access channels for 3 cannulas. One 12-mm trocar and two 5-mm trocars were passed through the port. The first trocar was placed with a laparoscope inside for visual guidance. The other trocars were then inserted under laparoscopic guidance from within the necrotic cavity. Necrosectomy was performed using a 5-mm laparoscope and 5-mm instruments. The perirenal fascia was opened along the front of the psoas muscle from the retrocolic space, and then the retroperitoneal space was opened along the top of the pancreatic tail until the lesser sac. Using warm saline positive pressure rinse and negative pressure aspirator to aspirate necrotic pus.

Single-hole forceps were used to grasp the solid necrotic tissue attached to the abscess, which was not easy to wash. Stop the operation and suture the incision when the abscess wall turns pink and there is no residual necrotic tissue or active hemorrhage. Our technique of retroperitoneoscopic pancreatic necrosectomy using the single-access port has several advantages. This technique allows safe placement of all working trocars under visual guidance and secure establishment of gas insufflation. The visualization of the necrotic cavity can be achieved using optional gas insufflation or continuous saline irrigation. Two additional instruments can be used simultaneously with a laparoscope. A 12-mm trocar allows the removal of large pieces of necrotic material and efficacious lavage of the necrotic cavity. The necrosis located in the lesser sac can be easily approached with this technique (Figure 3).
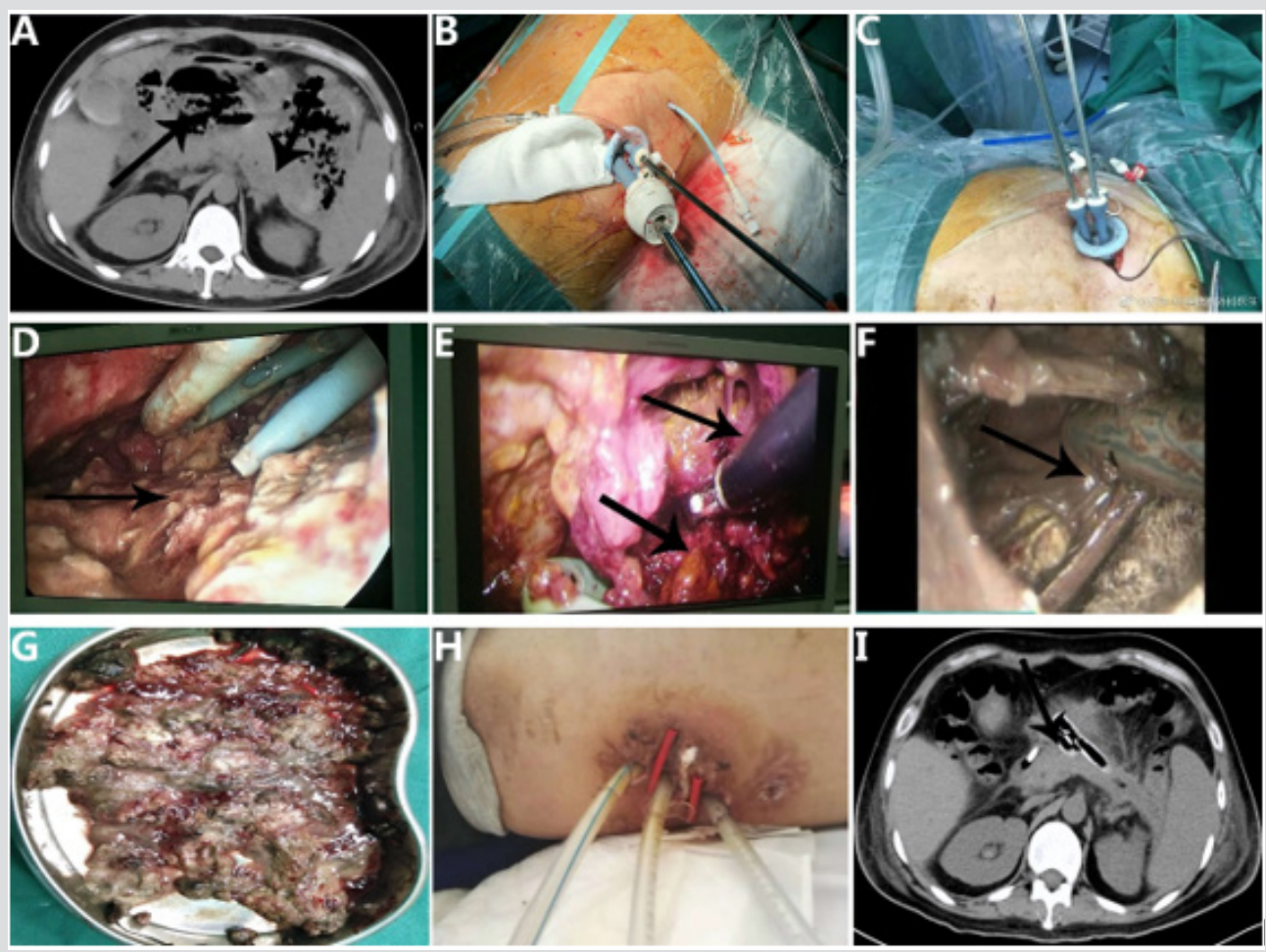

Figure 3: A: computed tomography before the first necrosectomy; B, C, D, E, and F: single-incision access port retroperitoneoscopic debridement; G: infected necrotic tissue; H: minimally invasive incision; I: computed tomography after last necrosectomy.

\section{Data Collection}

The following data were collected from the electronic files and patient charts: patient characteristics (age, gender, body mass index, Coexisting conditions), pancreatitis characteristics (etiology, Balthazar score, ASA class, CT severity index, Disease severity), The primary endpoint was a composite of major complications consist of treatment success, new-onset multiple organ failure, persistent systemic inflammatory response syndrome (SIRS), pancreaticcutaneous fistula, intra-abdominal bleeding and perforation of a visceral organ and death during 3 months of follow up. Secondary end points included pancreatic endocrine and exocrine insufficiency,
Incisional hernia, number of interventions, length of hospital stay (LOS), days in intensive care unit (ICU) and overall cost.

\section{Statistical Analysis}

For the statistical analysis, SPSS 22.0 software was used. The Kolmogorov-Smirnov test was used to assess whether continuous data were normally distributed. The results are presented as mean and standard deviations, as numbers and percentages or as median and percentiles when confidence intervals were too high. The qualitative variables were analysed with the chi-squared test and the continuous variables with the Kruskal-Wallis $\mathrm{H}$ test 
or linear-by-linear association tests. When comparing different methods of intervention, to reduce potential selection bias, we used multivariate logistic regression to adjust for prognostic baseline variables. These variables were CT severity index, American Society of Anesthesiologists (ASA) class, Acute Physiology and Chronic Health Evaluation II (APACHE II) score, and organ failure. Results of the adjusted analyses are presented as odds ratios with corresponding 95\% confidence intervals. Differences with $\mathrm{p}<0.05$ were accepted as statistically significant.

\section{Results}

Of the 1456 patients admitted to our hospital for AP, 1033 were diagnosed with MAP. Only 103 patients met the diagnostic criteria for INP.17 patients were excluded due to insufficient follow-up and 5 patients gave up treating due to lack of funds. Details of the 81 subjects were included in the study. Mean age was 45 years (range24-80) with a gender ratio of 1.3 (46 men and 35 women). The commonest etiologies in this series were biliary $(n=32,39 \%)$ and alcohol $(n=18,22 \%)$. Table 1 shows the baseline characteristics and disease severity for patients undergoing intervention, subdivided in 4 different minimally invasive approaches. No significant differences were found among the four groups regarding to patient features. As compared with patients treated PCD, patients undergoing MARPN, SIPN, and SIAPRD had higher ASA class, APACHE II score, CRP, WBC, CT severity index, single organ failure. But there were no significant differences in SIRS and multiple organ failure between the four groups.

Table 1: Characteristics of all patients with necrotizing pancreatitis.

\begin{tabular}{|c|c|c|c|c|c|}
\hline Characteristic & $\operatorname{PCD}(n=32)$ & MARPN(n=18) & $\operatorname{SIPN}(n=16)$ & SIAPRD(n=15) & $P$ value \\
\hline Age (y)(IQR) & $41(32-53)$ & $47(34-58)$ & $52(42-54)$ & $47(33-59)$ & 0.52 \\
\hline Male sex (\%) & $17(53 \%)$ & $11(62 \%)$ & $10(64 \%)$ & $8(55 \%)$ & 0.89 \\
\hline $\begin{array}{c}\text { Etiology (\%) } \\
\text { Gallstones } \\
\text { Alcohol abuse } \\
\text { Other } \\
\text { Unknown }\end{array}$ & $\begin{array}{c}13(40 \%) \\
7(22 \%) \\
5(17 \%) \\
7(22 \%)\end{array}$ & $\begin{array}{l}7(39 \%) \\
4(22 \%) \\
3(17 \%) \\
4(23 \%)\end{array}$ & $\begin{array}{l}6(38 \%) \\
4(25 \%) \\
3(19 \%) \\
3(19 \%)\end{array}$ & $\begin{array}{c}6(40 \%) \\
3(20 \%) \\
2(13 \%) \\
4(27 \%) \\
28(24-32)\end{array}$ & 0.87 \\
\hline BMI $\left(\mathrm{kg} / \mathrm{m}^{2}\right)(\mathrm{IQR})$ & $28(25-31)$ & $26(23-32)$ & $29(24-33)$ & & 0.84 \\
\hline ASA class (\%) & & & & & 0.03 \\
\hline I(healthy) & $3(9 \%)$ & $1(6 \%)$ & $1(6 \%)$ & $0(0 \%)$ & \\
\hline II (mild-systemic) & $11(34 \%)$ & $4(23 \%)$ & $3(19 \%)$ & $2(13 \%)$ & \\
\hline III (severe-systemic) & $18(56 \%)$ & $13(77 \%)$ & $12(81 \%)$ & $13(87 \%)$ & \\
\hline Coexisting conditions (\%) & & & & & 0.38 \\
\hline Cardiovascular disease & $15(47 \%)$ & $7(38 \%)$ & $6(38 \%)$ & $5(33 \%)$ & \\
\hline Pulmonary disease & $8(25 \%)$ & $5(28 \%)$ & $3(18 \%)$ & $4(27 \%)$ & \\
\hline renal insufficiency & $2(6 \%)$ & $1(6 \%)$ & $0(0 \%)$ & $1(7 \%)$ & \\
\hline Diabetes & $4(12 \%)$ & $3(17 \%)$ & $3(18 \%)$ & $3(20 \%)$ & \\
\hline CT severity index (\%) & & & & & $<0.01$ \\
\hline $0-2$ & $0(0 \%)$ & $0(0 \%)$ & $0(0 \%)$ & $0(0 \%)$ & \\
\hline 6-Apr & $6(18 \%)$ & $1(8 \%)$ & $1(9 \%)$ & $0(0 \%)$ & \\
\hline 10-Aug & $26(81 \%)$ & $12(92 \%)$ & $10(90 \%)$ & $9(100 \%)$ & \\
\hline \multicolumn{6}{|l|}{ Disease severity (\%) } \\
\hline SIRS & $29(91 \%)$ & $18(100 \%)$ & $16(100 \%)$ & $15(100 \%)$ & 0.18 \\
\hline ICU/high acuity care & $23(72 \%)$ & $16(89 \%)$ & $16(100 \%)$ & $15(100 \%)$ & 0.01 \\
\hline Single-organ failure & $10(31 \%)$ & $9(50 \%)$ & $10(62 \%)$ & $12(80 \%)$ & 0.01 \\
\hline Multiple organ failure & $7(22 \%)$ & $5(31 \%)$ & $7(45 \%)$ & $6(33 \%)$ & 0.37 \\
\hline Acute physiology score & $9(6-15)$ & $10(7-18)$ & $11(7-16)$ & $13(9-19)$ & 0.02 \\
\hline APACHE II score & $15(13-16)$ & $17(16-20)$ & $21(19-22)$ & $22(21-24)$ & $<0.01$ \\
\hline Glasgow score & $2(1-3)$ & $2(1-3)$ & $2(1-3)$ & $2(1-3)$ & 0.99 \\
\hline CRP (mg/L)(IQR) & 178(103-256) & $169(101-243)$ & 193(115-298) & 198(123-316) & 0.05 \\
\hline WBC (Í10) (IQR) & $14.6(10.3-18.7)$ & $15.3(10.9-20.1)$ & $14.3(10.1-17.9)$ & $15.9(11.4-21.8)$ & 0.27 \\
\hline
\end{tabular}

PCD, percutaneous catheter drainage. MAPRN, minimal access retroperitoneal pancreatic necrosectomy. SIPN, small incision pancreatic necrosectom. SIAPRD, single-incision access port retroperitoneoscopic debridement. BMI, body mass Index. ASA, American Society of Anesthesiologists. SIRS, systemic inflammatory response syndrome. APACHE, Acute Physiology and Chronic Health Evaluation. ICU, intensive care unit. CRP, C-reactive protein. WBC, white blood cell count. 
Table 2 lists primary endpoints and secondary endpoints for the several treatment approaches. In-hospital mortality was $18 \%$ overall, PCD has the greatest mortality (34\%) but there was no significant difference between other approaches (MARPN, 11\%; SIPN, 6\%; SIAPRD, 6\%). However, new onset organ failure occurred more frequently in the PCD (25\%) and MARPN (22\%) group. There was a significant difference in the improvement of sepsis after primary surgery (PCD 56\% vs MARPN 50\% vs SIPN 31\%vs
SIAPRD13\%; $\mathrm{P}<0.05)$. We observed no significant difference in pancreatic fisture, abdominal bleeding and visceral perforation among groups. Both ICU and Hospital stays were significantly longer in the patients undergoing PCD and MARPN for INP separately. Patients in the PCD, MARPN and SIPN groups required more number of interventions and higher treatment cost than SIAPRD $(\mathrm{P}<0.05)$. At 6 month follow-up, we observed no differences regarding exocrine, endocrine insufficiency and Incisional hernia.

Table 2: Clinical outcome of all patients with necrotizing pancreatitis.

\begin{tabular}{|c|c|c|c|c|c|c|c|}
\hline Characteristic & $\operatorname{PCD}(n=32)$ & MARPN(n=18) & $\operatorname{SIPN}(n=16)$ & SIAPRD(n=15) & $\begin{array}{c}\text { SIAPRDvs } \\
\text { PCD (P } \\
\text { value) }\end{array}$ & $\begin{array}{l}\text { SIAPRDvs } \\
\text { MARPN (P } \\
\text { value) }\end{array}$ & $\begin{array}{c}\text { SIAPRDvs } \\
\text { SIPN (P } \\
\text { value) }\end{array}$ \\
\hline \multicolumn{8}{|l|}{ Primary endpoint } \\
\hline $\begin{array}{c}\text { Treatment success* } \\
(\%)\end{array}$ & $15(46 \%)$ & $10(56 \%)$ & $15(93 \%)$ & $14(93 \%)$ & $<0.01$ & 0.01 & 0.96 \\
\hline Death (\%) & $11(34 \%)$ & $2(11 \%)$ & $1(6 \%)$ & $1(6 \%)$ & 0.04 & 0.66 & 0.96 \\
\hline SIRS (\%) & $18(56 \%)$ & $9(50 \%)$ & $5(31 \%)$ & $2(13 \%)$ & $<0.01$ & 0.02 & 0.23 \\
\hline \multicolumn{8}{|l|}{ Organ failure (\%) } \\
\hline $\begin{array}{c}\text { Pancreatic fistula } \\
(\%)\end{array}$ & $8(25 \%)$ & $4(22 \%)$ & $1(6 \%)$ & $0(0 \%)$ & 0.05 & 0.05 & 0.32 \\
\hline $\begin{array}{c}\text { Visceral } \\
\text { perforation (\%) }\end{array}$ & $1(3 \%)$ & $1(5 \%)$ & $3(18 \%)$ & $0(0 \%)$ & 0.48 & 0.35 & 0.07 \\
\hline $\begin{array}{c}\text { Abdominal } \\
\text { bleeding (\%) }\end{array}$ & $3(9 \%)$ & $0(0 \%)$ & $1(6 \%)$ & $1(6 \%)$ & 0.75 & 0.26 & 0.96 \\
\hline \multicolumn{8}{|l|}{$\begin{array}{c}\text { Secondary } \\
\text { endpoint }\end{array}$} \\
\hline $\begin{array}{c}\text { Incisional hernia } \\
(\%)\end{array}$ & $0(0 \%)$ & $1(5 \%)$ & $2(12 \%)$ & $1(6 \%)$ & 0.14 & 0.89 & 0.58 \\
\hline $\begin{array}{c}\text { Number. of } \\
\text { interventions(IQR) }\end{array}$ & $6(2-9)$ & $5(2-7)$ & $3(1-6)$ & $2(1-3)$ & 0.02 & 0.02 & 0.05 \\
\hline $\begin{array}{c}\text { New onset diabetes } \\
(\%)\end{array}$ & $4(12 \%)$ & $2(11 \%)$ & $3(18 \%)$ & $2(13 \%)$ & 0.93 & 0.84 & 0.68 \\
\hline $\begin{array}{c}\text { Exocrine } \\
\text { insufficiency (\%) }\end{array}$ & $5(15 \%)$ & $1(5 \%)$ & $2(12 \%)$ & $2(13 \%)$ & 0.83 & 0.43 & 0.94 \\
\hline $\begin{array}{l}\text { Length of ICU } \\
\text { stay(IQR) }\end{array}$ & $5(2-11)$ & $4(1-10)$ & $5(2-13)$ & $5(2-11)$ & 0.92 & 0.75 & 0.89 \\
\hline $\begin{array}{l}\text { Total hospital } \\
\text { stay(IQR) }\end{array}$ & $102(53-136)$ & $80(53-122)$ & $67(43-82)$ & $45(31-59)$ & 0.01 & 0.02 & 0.04 \\
\hline $\begin{array}{l}\text { Mean total } \\
\text { costs(IQR) }\end{array}$ & $\begin{array}{c}\text { 249698(193937- } \\
355173)\end{array}$ & $\begin{array}{l}\text { 198644(95916- } \\
327250)\end{array}$ & $\begin{array}{c}\text { 155374(86723- } \\
215381)\end{array}$ & $\begin{array}{c}119586(79716- \\
156733)\end{array}$ & $<0.01$ & 0.03 & 0.05 \\
\hline
\end{tabular}

*Within 30 days of intervention, patients survived and the clinical improvement. PCD, percutaneous catheter drainage. MAPRN, minimal access retroperitoneal pancreatic necrosectomy. SIPN, small incision pancreatic necrosectom. SIAPRD, single-incision access port retroperitoneoscopic debridement. SIRS, systemic inflammatory response syndrome. ICU, intensive care unit.

\section{Discussion}

Infection of necrosis occurred in approximately $30 \%$ of patients with necrotizing pancreatitis [15]. It is a heterogeneous disease with a high mortality rate [16]. Therefore, treatment must be individualized to specific patient characteristics, including necrosis distribution and size. Since Freeny et al. first reported the use of PCD in the treatment of INP [17], minimally invasive and endoscopic approach has gradually become the primary therapy in the management of INP [18]. And a recent meta-analysis of prospective studies of endoscopic approach in INP, demonstrated no remarkable superiority in the primary outcome compared with minimally invasive approach [19]. As yet, an ideal intervention has not been defined. There are few other studies that have directly compared different minimally invasive necrosectomy. Our multidisciplinary group consists of expert interventional radiologists and pancreatic surgeons. We utilized multiple approaches to NP treatment in this contemporary period. Mortality in infected necrosis in our study was $18 \%$. This seems to be lower than the mortality of approximately $30 \%$ for infected necrosis reported in reviews of the literature of the past 2 decades [20]. Mainly because we have improved and developed some new minimally invasive technologies. 
This study included a large number of patients in a relatively short study period, was conducted in our single centre setting, and covered the entire clinical spectrum of necrotizing pancreatitis. For a long time, we tried to improve PCD by selecting various penetrating sites and expanding the diameter of the puncture tube for continuous convection washing, but the clinical effect is not significant. Later, we began to try to improve the method of debridement surgery. SIPN, which we have improved on the basis of open surgical treatment, has minimal trauma, few local complications and high efficiency in removing necrotic tissue. This technique mostly removes necrotic tissue from the anterior abdominal cavity or retroperitoneum through a small incision along the PCD tube tract. Over the time period of this study, we found a necrotic resection method (SIAPRD) that is more novel and effective than SIPN. SIAPRD (a newest minimally invasive technique) was associated with fewer complications (organ failure, SIRS), and the use of SIAPRD instead of PCD, MARPN and SIPN as the first-line surgery method used to treat IPN was associated with shorter ICU and hospital stays and with shorter times on nutrition and hemodynamic support which may reflect an efficiency and safety greatly increased in removal of necrotic infection. And this method can maximally improve the systemic inflammatory state and minimize the trauma to the patient.

These results support that the significant decreased systemic and local inflammation with SIAPRD compared with other minimally invasive procedures, effectively control of infected necrotic lesions, thereby avoiding the need for multiple surgeries. In the other three groups, about $60 \%$ of patients need more than 2 necrotic resections to recover. Although endoscopic necrosis has been accepted, applied sparingly in our institutional experience, likely reflecting highly select indications, as well as liberal use of surgical transgastric debridement. In the past year, increasing number severe SAP have come to our centre for treatment. We prefer SIAPRD to treat patients and observe the superiority of this method. So far, only one patient has died, and length of hospital and hospitalization costs of all patients have been greatly reduced. We are conducting some prospective studies to further confirm the clinical efficacy of SIRLD. In our practice, SIAPRD acts a crucial therapeutic role in NP patients. Importantly, these approaches are individualized based on specific patient characteristics including necrosis distribution, physiologic condition, and failure to progress after other treatment. Over this time period we witnessed a significant increase in the number of unsuccessful NP patients treated with PCD and MARPN; the relatively high number that still require effective debridement $(>50 \%$ ) likely reflects the highly complex nature of patients referred to our tertiary centre.

These complex clinical scenarios include patients who have failed other therapies, as well as those with pancreatic head necrosis and necrosis tracking down paracolic gutters and the root of the small bowel mesentery. This report compares the characteristics and clinical outcomes of different treatments in our surgical centre and assess the best treatment options. From the current results analysis, SIAPRD is a very effective and safe method, and it is necessary to widely promote it. At the same time, it should be noted that necrotizing pancreatitis is a complex and heterogeneous disease. We are supposed to treat patients individually according to the degree of disease progression and the anatomical distribution of necrotic foci. Minimally invasive surgery is only a means, not a constant. Many patients will require more than one modality to effect disease resolution, and operative debridement continues to play an important role in management of these patients. Evaluation by a multidisciplinary treatment team composed of experienced gastroenterologists, surgeons, and interventional radiologists is crucial for treatment planning and to achieve optimal patient outcomes. One patient in the SIAPRD group died of abdominal bleeding on the 5 th day after surgery.

He was 82 years old, obese (BMI = 36), and had hypercholesterolemia, hypertension, and chronic cardiac failure. After 2 weeks of treatment in the external hospital, he continued to have high fever and shortness of breath and transferred to the surgical centre of our hospital for further treatment. At the time of admission, the patient had multiple venous thrombosis in both lower extremities, abnormal coagulation function, and the risk of surgery was extremely high. After communicating with the family, he decided to undergo surgical treatment. On the 5th day after the SIRLD, the patient developed abdominal bleeding and died after emergency laparotomy. Our study also has limitations, first, this is a retrospective study that utilized direct medical record, and while follow-up was assessed for the present time, there inherently may be recall bias with other details of the interview and history reporting. Second, this is a single centre study, which typically offers different therapies, our results may not represent other healthcare settings with differing patient demographics and procedural preferences, such as percutaneous retroperitoneal nephrostomy. Third, transgastric drainage is seldom used in our centre. Last but not least, a caveat is in order, however, as SIAPRD and SILD may have been used preferentially in the sickest patients during the late period that may be bias in analysing the prognosis of patients.

\section{Conclusion}

In summary, SIAPRD has obvious advantages in the treatment of INP, It is safe and effective and can greatly reduce hospitalization time and cost. This study was retrospective, and the sample size was small. So, there is a great need for more RCTs to confirm these advantages. In addition, future studies will be required to further define the optimal time for the SIAPRD procedure.

\section{Acknowledgement}

This study was funded by Key project to tackle key problems in health industry in Tianjin 16KG159, Project of Tianjin thousands of people plan. 


\section{References}

1. Portelli M, Jones CD (2017) Severe acute pancreatitis: pathogenesis, diagnosis and surgical management. Hepatobiliary Pancreat Dis Int 16(2): 155-159.

2. Shyu JY, Sainani NI, Sahni VA, Chick JF, Chauhan NR, et al. (2014) Necrotizing pancreatitis: diagnosis, imaging, and intervention. Radiographics 34 (5): 1218-1239.

3. Lankisch PG, Apte M, Banks PA (2015) Acute pancreatitis. Lancet 386(9988): 85-96.

4. Peery AF, Crockett SD, Barritt AS, Dellon ES, Eluri S, et al. (2015) Burden of Gastrointestinal, Liver, and Pancreatic Diseases in the United States. Gastroenterology 149(7): 1731-1741.

5. Pezzilli R, Zerbi A, Di Carlo V, Bassi C, Delle Fave GF, et al. (2010) Practical guidelines for acute pancreatitis. Pancreatology 10(5): 523-535.

6. Roch AM, Maatman T, Carr RA, Easler JJ, Schmidt CM, et al. (2018) Evolving treatment of necrotizing pancreatitis. Am J Surg 215(3): 526529.

7. Whitcomb DC (2006) Clinical practice. Acute pancreatitis. N Engl J Med 354: 2142-2150.

8. Zerem E (2014) Treatment of severe acute pancreatitis and its complications. World J Gastroenterol 20(38): 13879-13892.

9. Boumitri C, Brown E, Kahaleh M (2017) Necrotizing Pancreatitis: Current Management and Therapies. Clin Endosc 50(4): 357-365.

10. Van Santvoort HC, Besselink MG, Bakker OJ, Hofker HS, Boermeester MA, et al. (2010) A step-up approach or open necrosectomy for necrotizing pancreatitis. N Engl J Med 362: 1491-1502.

11. Van Brunschot S, van Grinsven J, van Santvoort HC, Bakker OJ, Besselink MG, et al. (2018) Endoscopic or surgical step-up approach for infected necrotising pancreatitis: a multicentre randomised trial. Lancet 391(10115): 51-58.

\section{ISSN: 2574-1241}

DOI: 10.26717/BJSTR.2019.18.003100

Yunfeng Cui. Biomed J Sci \& Tech Res

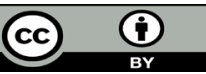

This work is licensed under Creative Commons Attribution 4.0 License

Submission Link: https://biomedres.us/submit-manuscript.php
12. Banks PA, Bollen TL, Dervenis C, Gooszen HG, Johnson CD, et al. (2013) Classification of acute pancreatitis 2012: revision of the Atlanta classification and definitions by international consensus. Gut 62(1): 102-111.

13. Pezzilli R, Zerbi A, Campra D, Capurso G, Golfieri R, et al. (2015) Consensus guidelines on severe acute pancreatitis. Dig Liver Dis 47(7): 532-543.

14. Knaus WA, Sun X, Nystrom O, Wagner DP (1992) Evaluation of definitions for sepsis. Chest 101(6): 1656-1662.

15. Van Dijk SM, Hallensleben NDL, Van Santvoort HC, Fockens P, Van Goor $\mathrm{H}$, et al. (2017) Acute pancreatitis: recent advances through randomised trials. Gut 66(11): 2024-2032.

16. Morato O, Poves I, Ilzarbe L, Radosevic A, Vazquez-Sanchez A, et al. (2018) Minimally invasive surgery in the era of step-up approach for treatment of severe acute pancreatitis. Int J Surg 51: 164-169.

17. Freeny PC, Hauptmann E, Althaus SJ, Traverso LW, Sinanan M (1998) Percutaneous CT-guided catheter drainage of infected acute necrotizing pancreatitis: techniques and results. AJR Am J Roentgenol 170(4): 969975.

18. Fagenholz PJ, Thabet A, Mueller PR, Forcione DG (2016) Combined endoscopic trangastric drainage and video assisted retroperitoneal pancreatic debridement - The best of both worlds for extensive pancreatic necrosis with enteric fistulae. Pancreatology 16(5): 788-790.

19. Gurusamy KS, Belgaumkar AP, Haswell A, Pereira SP, Davidson BR (2016) Interventions for necrotising pancreatitis. Cochrane Database Syst Rev 4: Cd011383.

20. Da Costa DW, Boerma D, van Santvoort HC, Horvath KD, Werner J, et al. (2014) Staged multidisciplinary step-up management for necrotizing pancreatitis. Br J Surg 101(4): e65-79.

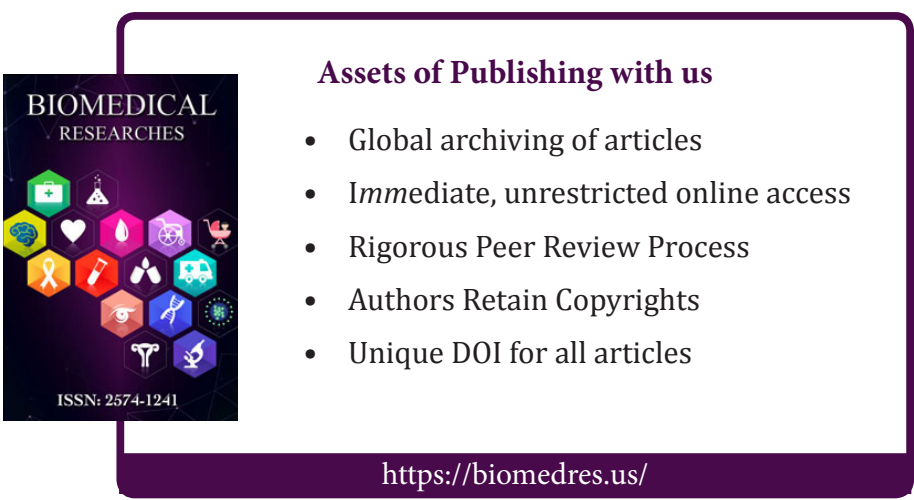

